\title{
Thoracolumbar Fracture Dislocation: A Study of 30 Patients
}

\author{
H. Kinoshita, MD ${ }^{1}$, Y. Nagata, $\mathbf{M D}^{1}$, H. Hirakawa, $\mathbf{M D}^{2}$ \\ ${ }^{1}$ Department of Orthopedic Surgery, Chugoku Rosai Hospital, 1477 Hiromachi, Kure \\ 737-01, Japan, ${ }^{2}$ Department of Orthopedic Surgery, Higashi Nagano National \\ Hospital, 2-477 Ueno, Nagano 380, Japan.
}

\section{Summary}

Open reduction and wiring of the spinous processes were carried out in patients with a thoracolumbar fracture dislocation. Clinical and $X$-ray examinations were performed on 30 patients 5 or more years following operation.

At the time of the initial examination, an anterior angulation of $30^{\circ}$ or more was noted in 18 patients, but only in 4 cases at the time of the follow-up examination. Anterior displacement of the vertebral bodies was seen in 30 patients, but it was found to be normal in 26 patients at the time of follow-up. There were 7 patients with lateral angulation of $5^{\circ}$ or more, but only 2 patients at the time of follow-up. Lateral displacement of $5 \mathrm{~mm}$ or more was observed in 10 patients, but only 3 patients could be seen at the time of follow-up.

In comparison with spinal instrumentation with the use of large long metallic materials, wiring of the spinous processes has several advantages such as limited surgical invasion, firm fixation, no effect on spinal mobility and no need for repeat surgery for removal of metallic materials. It is an adequate alternative method for the internal fixation of rotational fracture dislocations of the thoracolumbar region of the spine, without fracture of the posterior wall of the vertebral body and pedicle fracture.

Key words: Fracture dislocation; Thoracolumbar spine; Spinal surgery; Outcome.

In recent years Harrington rods and Luque rods have been extensively employed in the operative fixation of vertebral fractures and fracture dislocations, but slipped hooks, broken rods, and loss of correction are not uncommon (Dick, 1984), and the long length of immobilisation of healthy joints has presented rehabilitation problems (Guttmann, 1969).

Stanger (1947), Roger (1957), and Kaufer (1966) have bound the spinal processes with wire for the internal fixation of vertebral fracture dislocation and obtained satisfactory results, but hardly any reports have been published in the 
literature on a large number of cases. The authors have employed this method consistently from 1965 and the indications, operative procedures, and therapeutic results are presented in this report.

\section{Materials and methods}

Thirty patients are presented: 27 males and 3 females. The surgical procedure was conducted from 1965 to 1980 at the Chugoku Rosai Hospital. The age ranged from 19 to 60 years with an average age of 33.7 years. The sites of the fracture dislocation were located below the eleventh thoracic vertebra including T12, L1 and L2 vertebrae (Table).

\begin{tabular}{lr} 
Table & \\
& \\
\hline T11/12 & 8 \\
T12/L1 & 17 \\
L1/L2 & 5 \\
& 30 \\
\hline
\end{tabular}

The roentgenographic findings at the time of the initial examination showed a rotational fracture dislocation in 29 patients with the upper vertebra swinging around the lower vertebral body to carry with it the intervertebral disc and a wedged slice of the upper border of the lower vertebral body and an associated facet fracture in 10 patients, a pedicle fracture in 2 patients (right pedicle fracture of T12 in 1, and a left pedicle fracture of L2 in 1), and a transverse process fracture in 22 patients. In 1 patient there was severe dislocation of L1/L2 with a fracture of facets and transverse processes (Fig. 1).

The degree of the anterior displacement of the vertebral body was classified as grade 1 when there was a displacement of $25 \%$ of the vertebral body width, as grade 2 for displacement of $50 \%$, grade 3 for displacement of $75 \%$ and

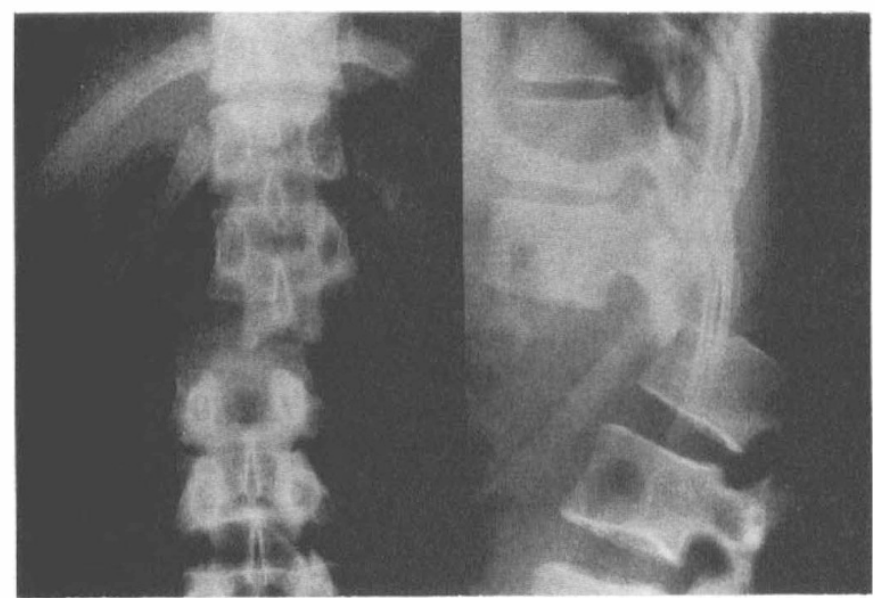

Figure 1 Dislocation of grade 4 of $\mathrm{L} 1 / 2$ accompanied by fracture of the bilateral superior articular and transverse processes of $\mathrm{L} 2$. 
grade 4 for displacement of $100 \%$ or more. Five cases belonged to grade 1, 22 to grade 2, 2 to grade 3 and 1 to grade 4 . Fourteen patients had associated lateral displacement.

Concerning the neural deficit, 19 patients had complete paraplegia, 5 had nerve root escape, $3 \mathrm{had}$ an incomplete paraplegia, and in 3 patients there was no neural deficit.

The time lapse from injury to surgery was within 48 hours in 18 patients, within 1 week in 4 , within 2 weeks in 7 , and more than 2 weeks in 1 patient.

The operation was performed with the patient in the prone position with the head and lower extremities placed in a sling for hyperextension of the spine. Under local or general anaesthesia, the site of injury was exposed and a hook was placed on the intact neural arch superior and inferior to the injured site. While applying traction, the posteriorly dislocated upper facets of the lower vertebra were repositioned using an elevator for leverage, and the dislocation was carefully reduced. It was sometimes necessary to apply gentle flexion to the spine to remove the locked facet, but in only 1 case was it necessary to carry out a partial resection of the facet. After reducing the facet displacement, the patient's head was fixed in position and the lower extremities were pulled upwards. With hyperextension of the spine, the kyphotic angulation was corrected and the vertebral fracture was reduced.

A transverse hole was made through the base of the spinous process above the level of the dislocation. A $1 \mathrm{~mm}$ diameter soft stainless steel wire was placed through this hole and looped under the spinous process of the segment below the dislocation and then drawn firmly together.

In the first 8 cases, a bone graft was applied to the lamina for posterior spinal fusion. However, since 1970 only the spinous processes were bound by stainless wire, without posterior bony fixation.

After the operation the patient is placed on a Stryker turning frame to prevent the development of bed sores, and after the eighth week the patient is transferred to a regular bed and sitting training is commenced.

From the tenth to thirteenth week, standing training using a standing table and partial weight-bearing training are initiated, and after confirming roentgenographically that vertebral fixation is secure, mat training is begun.

\section{Case 1}

A 28-year-old male who sustained an anterolateral fracture dislocation of the first lumbar vertebra, fracture of the bilateral superior articular process of L2, the right transverse process of $\mathrm{L} 1$ and the transverse processes of $\mathrm{L} 2$ and $\mathrm{L} 3$ when a forklift fell on his back (Fig. 1). On the day following the injury, under general anaesthesia reduction was carried out and the spinous processes were wired. A bone graft was not used. The findings of the roentogenogram taken 11 years after the operation showed osseous fusion of the vertebral bodies with anterior angulation of $20^{\circ}$ without retranslocation (Fig. 2).

\section{Case 2}

A 43-year-old male fell and sustained a fracture dislocation between $\mathrm{T} 1$ and $\mathrm{T} 2$. On the following day the spinous processes of $\mathrm{T} 10$ and $\mathrm{T} 12$ were wired under general anaesthesia (Fig. 3). Roentgenographic findings 20 years after surgery showed an anatomical cure but without osseous fusion of the vertebral bodies (Fig. 4). 


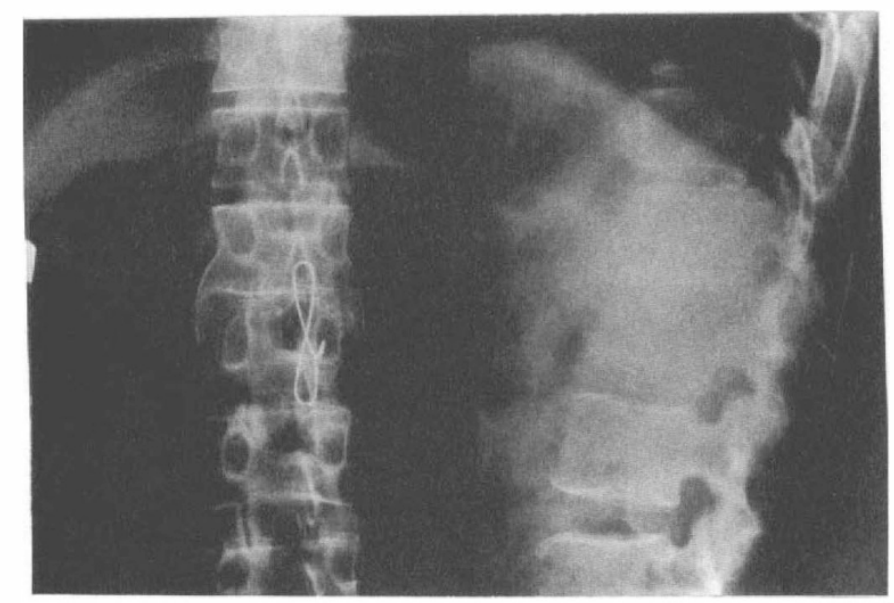

Figure 2 Same case as in Figure 1, 11 years after surgery. Osseous fusion of vertebral bodies with $20^{\circ}$ anterior angulation.

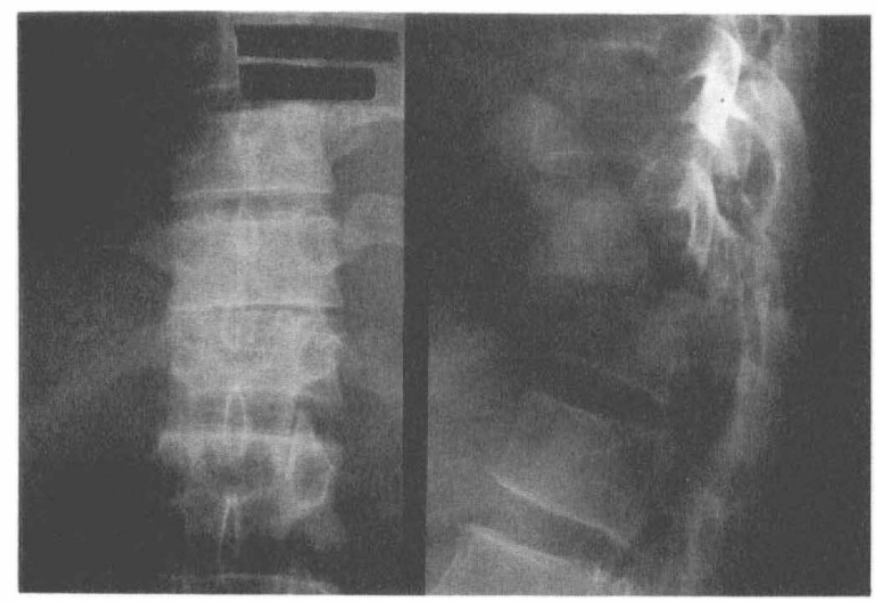

Figure 3 Fracture dislocation of T11/12 of grade 2.

\section{Results}

Of a total of 37 patients with a thoracolumbar fracture dislocation, clinical and $\mathrm{X}$-ray examinations were carried out on 30 patients more than 5 years after the operation.

At the time of initial examination, a kyphotic angulation of $30^{\circ}$ or more was observed roentgenographically in 18 patients, but in only 4 at the time of the follow-up examinations. In 26 out of 30 patients the anterior displacement became normal with a dislocation of grade 1 in 3 patients and grade 2 in 1 patient. Among these there was 1 patient with a pedicle fracture of L2 (left). There were 7 cases of lateral angulation of $5^{\circ}$ or more, but only 2 could be observed at the time of follow-up. Lateral displacement of $5 \mathrm{~mm}$ or more had been observed in 10 patients, but only 2 could be seen at follow-up. One case of lateral displacement was noted at the time of follow-up though not evident at the time of the initial examination. In this patient the left pedicle fracture of T12 

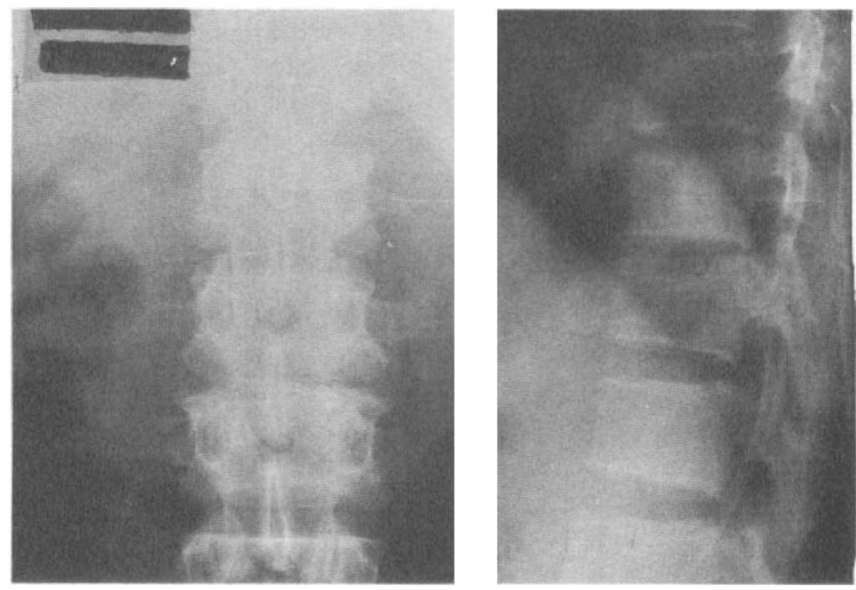

Figure 4 Same case as in Figure 3, 20 years after surgery. Anatomically cured but without fusion of the vertebral bodies.

had been observed. Our clinical results showed that the stability and mobility of the spine were satisfactory in all of our patients which were examined 5 or more years after surgery, but 4 complained of back pain on anteflexion and during cold weather though not of a degree to affect daily living. There was no case in which paraplegia developed or was exacerbated by the operation. There was no change in the paraplegia of the 19 patients with complete paraplegia. Regarding incomplete paraplegia and root escape, 1 patient belonged to grade B of Frankel's classification and 7 belonged to grade C. Following the operation 1 case improved to grade $\mathrm{D}$ and the $\mathrm{C}$ grade improved to grade $\mathrm{D}$. In the early stage 2 patients were observed who developed small pressure ulcers in the sacral region, but none were seen after 1970 .

\section{Discussion}

The aim of the treatment of fracture dislocations of the thoracolumbar spine is to restore and/or maintain alignment and stability in preparation for future weight-bearing (Hardy, 1965). Postural reduction is regarded to be the best procedure (Guttmann, 1969, 1976; Frankel, 1969). There is however, a risk of neural damage if the dislocated spine becomes excessively extended with the facet locked (Roger, 1938; Kaufer, 1966).

The authors exposed the injured site and at operation reduced the dislocated facet under good visual control.

In flexion-rotation injury, the posterior ligament complex is ruptured and stability is lost (Holdsworth, 1953). In the present series, the posterior ligament complex was ruptured in all the patients. By binding the spinous processes with steel wire, the function of the posterior ligament complex could be restored, and by converting the unstable fracture dislocation into a stable fracture dislocation, it was possible to eliminate the tendency to redisplacement. As almost all the cases could be cured without redisplacement except those with fracture dislocation accompanied by a pedicle fracture $(6.6 \%$ in the present series), 
the role played by the posterior ligament complex in the stability of the spine, as reported by Holdsworth (1953, 1963, 1970), cannot be over-emphasised.

It was discovered in 8 patients that the wire was broken 8.5 weeks to 10 months after the operation, but there was no recurrent case of displacement because the wire was broken.

Following the operation, the patient is placed on a Stryker frame and the development of pressure ulcers is prevented by changing the body position. By binding the spinous processes with steel wire, the injured site becomes stable and the pain alleviated, facilitating nursing management. From the ninth week, sitting training is commenced. The time spent in bed is not wasted as physiotherapy and occupational therapy prepare the patient both physically and mentally for the next stage of rehabilitation.

Osseous fusion between the reduced upper vertebral bodies and vertebral bodies below observed roentgenographically at the time of follow-up was confirmed in 19 patients (13 cases of bridgebuilding and 6 cases of blockbuilding) and osteophyte formation without osseous fusion or absence of osteophyte formation in 11 patients, but no patient showing instability or restricted mobility was observed.

No difference could be demonstrated clinically and roentgenographically between patients with a bone graft and patients without a bone graft. It was thus found that from the standpoint of reducing operative invasion, posterior fixation is not necessary.

\section{Conclusion}

1. In all the cases, atraumatic reduction was possible with no case of exacerbation of postoperative neutral deficit.

2. Firm fixation was possible by only wiring of the spinous processes, with recurrent dislocation developing in only 2 patients with unilateral pedicle fracture.

3. It was observed that the fixing wire became broken in 8 patients, but in none did it bring about deformation or translocation.

4. In this procedure, surgical invasion is minimal with no effect on spinal mobility except for the injured segment and no repeat surgery for the removal of metal fixation was necessary.

5. Nursing management such as changing of body position during the postoperative period is facilitated.

6. By this procedure, spinal deformation could be corrected and satisfactory stability could be restored, but it was ineffective in alleviating paralysis.

7. In recent years, spinal instrumentation with the use of large long metallic materials is widely employed, but it is considered that the procedure discussed above, involving minimal surgical invasion but providing satisfactory results, can be strongly recommended.

\section{References}

Dick W, KLuger P, MAgerl F, et al. 1985 A new device for fixation of thoracolumbar and lumbar spine fractures: The 'fixateur interne.' Paraplegia 22:225-232. 
Frankel H, HANCOCK, DO, Hyslop G, et al. 1969 The value of postural reduction in the initial management of closed injuries of the spine with paraplegia and tetraplegia. Paraplegia 7:179-192.

GUTTMANN L 1969 Spinal deformaties in traumatic paraplegics and tertraplegics following surgical procedures. Paraplegia 7:38-49.

GutTMANN L. 1976 Spinal cord injuries. In: Comprehensive Management and Research, Blackwell Scientific Publications, Oxford, London, Edinburgh and Melbourne, pp. 142-148.

HARDY AG 1965 The treatment of paraplegia due to fracture-dislocations of the dorso-lumbar spine. Paraplegia 3:112-123.

HOLDSWORTH FW, HARDY A. 1953 Early treatment of paraplegia from fractures of the thoracolumbar spine. Fournal of Bone and Foint Surgery 35B:540-550.

HOLDSWORTH FW 1963 Fractures, dislocations, and fracture dislocations. fournal of Bone and Foint Surgery 45B:6-20.

HOLDSWORTH FW 1970 Fractures, dislocations, and fracture dislocations of the spine. Fournal of Bone and foint Surgery 52A:1534-1551.

KAUFER H. 1966 Lumbar fracture-dislocation. A study of twenty-one cases. Fournal of Bone and foint Surgery 49A:712-730.

ROGERS WA 1938 Cord injury during reduction of thoracic and lumbar vertebral-body fracture and dislocation. Fournal of Bone and foint Surgery 20:689-695. 